\title{
ANALYSIS OF THE ANISOTROPY OF POINT DEFECT DIFFUSION IN HCP Zr
}

\author{
G.D. Samolyuk ${ }^{1}$, A.V. Barashev ${ }^{1,2}$, S.I. Golubov ${ }^{1}$, Y.N. Osetsky ${ }^{1}$, and R.E. Stoller ${ }^{1}$ \\ ${ }^{1}$ Materials Science and Technology Division, ORNL, Oak Ridge, TN 37831- 6138, USA \\ ${ }^{2}$ Center for Materials Processing, Department of Materials Science and Engineering, \\ University of Tennessee, East Stadium Hall, Knoxville, TN 37996-0750, USA
}

\begin{abstract}
A combination of density functional theory (DFT), kinetic Monte Carlo and mean-field rate theory is applied to analyze point defect migration and its effect on the observed growth of hcp $\mathrm{Zr}$ under $1 \mathrm{MeV}$ electron irradiation. DFT is used to study stability of various configurations of vacancies and self-interstitial atoms (SIAs) and migration barriers. The data are used in kinetic Monte Carlo modelling of defect diffusion at different temperatures. It is found that both defects exhibit anisotropic diffusion, predominantly parallel to the basal planes. The ratio of diffusion coefficients parallel and perpendicular to the basal planes is found to be higher for vacancies as compared to SIAs at temperatures below $\sim 600 \mathrm{~K}$. This raises doubts that the observed radiation growth in $\mathrm{Zr}$ irradiated with $1 \mathrm{MeV}$ electrons, namely positive strains in prismatic and negative strains in basal directions, and void alignment along basal planes, can be accounted for by the anisotropy of point defect diffusion, which predicts opposite strain signs. It is speculated that formation of small SIA clusters with higher diffusion anisotropy may be responsible for the experimental observations.
\end{abstract}




\section{INTRODUCTION}

Zirconium alloys are widely used in the nuclear industry because of their low absorption cross-section for neutrons. Neutron irradiation of Zr-based materials leads to radiation growth: expansion in the prismatic ( $a$ axes) and shrinkage in the basal ( $c$ axis) directions, and this is one of the major concerns for the safe operation of commercial light-water reactors. It has been shown $[1,2]$ that the observations can be accounted for by assuming the major mechanism of radiation growth to be associated with the cascade-produced one-dimensionally (1-D) mobile clusters of self-interstitial atoms (SIAs). The formation of SIA clusters in high energy cascades [3-5] and their 1-D mobility along the close-packed directions of basal planes [6] has been confirmed by molecular dynamics simulations in $\mathrm{Zr}$.

The radiation growth of $\mathrm{Zr}$ is also observed under $\sim 1 \mathrm{MeV}$ electron irradiation, where no displacement cascades are created and the primary damage is to be believed in the form of Frenkel pairs, i.e. single vacancies and SIAs. One possible explanation for this is to assume that single SIAs also exhibit diffusion anisotropy predominantly parallel to the basal planes. . This was, indeed, the main assumption of the Diffusion Anisotropy Difference (DAD) theory $[7,8]$. However, there are no experimental data confirming such diffusional properties of SIAs. Only limited molecular dynamics modelling of the diffusion in $\mathrm{Zr}$ has been carried out [9], although there are some experimental data $[10,11]$ on vacancy diffusion, demonstrating anisotropy of diffusion along the basal planes, i.e. similar to that assumed for the SIAs in DAD theory. Since damage accumulation is a result of the difference in vacancy and SIA absorption by various dislocations, the validity of DAD model should be examined.

The existence of the diffusion anisotropy in $\mathrm{Zr}$ under $1 \mathrm{MeV}$ electron irradiation is indicated by the observed void alignment in rows parallel to the basal plane [12]. According to Foreman [13], the alignment is associated with mutual screening of defects from mobile interstitial defects, and a more rigorous mathematical analysis [14] supports this. In the case of neutron irradiation this phenomenon can be explained by the well-known 1-D mobility of cascade-produced SIA clusters, whereas the alignment mechanism under electron irradiations is 
not yet clear. Taking into account that experimental measurements of SIA diffusion are not possible, the only way to clarify this mechanism is by multi-scale modelling.

Estimating diffusion anisotropy requires knowledge on stability of various defect configurations and activation energies for transitions between states. There have been several $a b$ initio studies of diffusion in $\mathrm{Zr}$ using the density functional theory (DFT) approach [15-23]. Vacancies were studied in a relatively small super-cell of $3 \times 3 \times 2$ [17] and significant anisotropy of single vacancy jumps was found. Accurate description of single SIAs requires larger supercells, hence more expensive calculations. Earlier studies used super-cells (e.g. $4 \times 4 \times 3$ in $[15,16])$ and predicted the symmetric octahedral "O" configuration (see Fig.1) to be the most stable, which should execute 3-D random walk. Further studies [18-23] demonstrated that much larger super-cells $(6 \times 4 \times 3$ or bigger) are required to obtain the correct hierarchy of SIA configurations. For these super-cells, the lowest energy configuration was basal octahedral (BO) with the basal split BS next lowest in energy (see Fig. 1). One configuration, $\mathrm{BC}^{\prime}$ [22], was found to have energy between those of $\mathrm{BO}$ and $\mathrm{BS}$, and to be separated from the BO by a small barrier, so it is unstable even at low temperatures [22]. The BO to BS barrier is slightly lower than that for BO to $\mathrm{O}$ [23]. To conclude, a more detailed study is required to obtain a complete description of point defect migration barriers. In addition, the use of a larger simulation cell may provide more accurate description of the energy barriers.

In the present study we use the DFT and kinetic Monte Carlo to analyze vacancy and SIA migration properties. A mean-field rate theory model is then applied to analyze the possibility of explaining radiation growth of hep $\mathrm{Zr}$ and void alignment under $1 \mathrm{MeV}$ electron irradiation. The paper is organised as follows. Section II contains the DFT computational details, together with estimation of the accuracy of the approach by comparing calculated lattice parameters and elastic constants with experimental values. The SIA formation energies are discussed and compared with the results in [22] in Section III. The results on the SIA and vacancy migration barriers are presented and discussed in Sections IV and V, respectively. In Section VI, the kinetic MC simulations of point defect diffusion are presented, and the possibility of explaining the observed growth strains is estimated on the basis of the DAD theory [8]. The conclusions are drawn in Section VII. 


\section{COMPUTATIONAL APPROACH}

The following observations from previous studies were taken into consideration when choosing the approach for this work: a) the generalized gradient approximation (GGA) reproduces the experimental $c / a$ ratio reasonably well; b) pseudo-potentials with smaller cores should be more appropriate due to possible core overlap in the SIA studies, and c) the SIA properties are sensitive to the super-cell size.

Electronic structure calculations were performed using the Quantum Espresso (QE) package [24] within the framework of DFT with the GGA approximation for the exchangecorrelation (XC) energy. The Perdew, Burke and Ernzerhof (PBE) [25] XC functional was used. The calculations were performed using a plane-wave basis set and an ultra-soft pseudo-potential [26,27]. A more detailed description of the pseudo-potentials used can be found in [15]. The formation energies were calculated using super-cells of $4 \times 4 \times 3$ unit cells for vacancies and $6 \times 4 \times 3$ for SIAs, containing 95 and 145 atoms, respectively. A plane-wave energy cut-off of 26 Ry was used in the QE calculations. The Brillouin zone (BZ) summation was carried out over $4 \times 4 \times 4$ or $3 \times 4 \times 4$ BZ grids for $4 \times 4 \times 3$ and $6 \times 4 \times 3$ super-cells, respectively, and the structure was relaxed until the energy convergence of $0.0015 \mathrm{eV}$ was reached. Following previous calculations [15], the super-cell dimensions containing an SIA (a vacancy) were scaled isotropically by a factor $[(N+1) / N]^{1 / 3}\left([(N-1) / N]^{1 / 3}\right.$ for vacancy), where $N$ is the number of atoms in a perfect super-cell. Such a procedure allowed the pressure to be kept close to zero with accuracy better than 1 kbar. The nondiagonal stress components were below 5 kbar. As demonstrated by Domain and Legris [16], the nonisotropic and isotropic cell relaxations produce the same sequence of SIA configurations. Some of the QE results on the SIA formation energy were verified using VASP package [28] calculations with the Perdew, Wang (PW) [29] XC functional and a plane-wave energy cut-off of $16.5 \mathrm{Ry}$.

The energy barriers for the SIA jumps were calculated using the nudged elastic band (NEB) method [30-32], as implemented in the QE code. Five intermediate states were calculated between each initial and final configuration, which were local minima singled out from the lowest-energy structures found. The super-cells were the same as for formation energy calculations. The search for the minimum energy path was terminated when the norm of the force orthogonal to the path became smaller than $0.01 \mathrm{eV} / \AA$. 
The approach used allowed reasonable accuracy to be achieved for the lattice parameter $a$ and elastic constants. Our results together with those in $[15,33,34]$ are collected in Table 1 . The local density approximation (LDA) with the Perdew, Zunger (PZ) [35] XC functional is known to systematically underestimate $a$ and overestimate $c / a$ in the pseudo-potential type calculations [15]. Similar to the previous observations [15,33,34], GGA calculations were found to reproduce the experimental value of $a$ and gives $c / a$ to be equal to 1.6 , what is very close to experimental value of 1.593. It should be mentioned that a $c / a$ of 1.585 , what is smaller than the experimental value, was obtained using full potential linear muffin tin orbitals method, while pseudo-potential methods give slightly larger values. Most elastic constants were calculated within $10 \%$ of experimental values, with the exceptions of $\mathrm{C}_{44}$ and $\mathrm{C}_{66}$, which were underestimated by 28 and $18 \%$, respectively. The latter discrepancy may be related to the sensitivity of these constants to details of electronic structure and/or to significant contribution of the atomic relaxation [15].

\section{SIA FORMATION ENERGIES}

The goal of the present study was to calculate the migration energy barriers for the most important SIA configurations in $\mathrm{Zr}$ : BO, BS, $\mathrm{O}$ and $\mathrm{S}$ (Fig. 1). In this section we summarize the formation energies of these configurations obtained here and compare with the published results.

The results are collected in Table 2 and use the $\mathrm{O}$ configuration as the reference state. The ground state corresponds to the $\mathrm{BO}$ configuration in all calculations with sufficiently large simulation cell. The next most stable is $\mathrm{BC}^{\prime}$ configuration, which is only $0.05 \mathrm{eV}$ higher in energy and is very close to the BO (see details in [22]), see Table 2. This configuration was obtained from the basic crowdion configuration by displacing its central atom towards one of the neighbouring octahedral sites. It was found to be $0.09 \mathrm{eV}$ higher than that of $\mathrm{BO}$ with atomic orbitals as the basis wave function (SIESTA) and $0.03 \mathrm{eV}$ higher with the plane-wave basis (VASP). (Note that the difference in the modelling cell size in these calculations might contribute to the energy difference.) In our calculations, the $\mathrm{BC}^{\prime}$ configuration was obtained as an intermediate metastable state on the path from $\mathrm{O}$ to $\mathrm{S}$. Another metastable $\mathrm{M}$ configuration was detected during $\mathrm{O}$ to BS transition, which was close both energetically and geometrically to 
$\mathrm{BC}^{\prime}$, as illustrated in Fig. 2. There are three equivalent $\mathrm{M}$ configurations in each basal-plane equilateral triangle formed by three nearest atoms in its corners. The interstitial atom in any of these configurations is shifted by $0.2 \AA$ along the line connecting the triangle center and one of the corner atoms away from the corner atom and by $0.4 \AA$ in $c$ direction (Fig. 2). The energy of $\mathrm{M}$ configuration is only $0.02 \mathrm{eV}$ higher than that for $\mathrm{BC}^{\prime}$, and it looks like the $\mathrm{BO}$ configuration with the central atom slightly shifted. Both $\mathrm{BC}^{\prime}$ and $\mathrm{M}$ configurations are very close to each other in energy and structure, and deserve special attention. As obtained in [23], the $\mathrm{BC}^{\prime}-\mathrm{O}$ migration barrier is very small and the saddle point almost disappears with a larger simulation cell. It was concluded that the $\mathrm{BC}^{\prime}$ is not a stable configuration [23] and corresponds to an extended flat energy surface. Since a similar small barrier of $0.008 \mathrm{eV}$ was obtained for the MBO transition (see Fig. 3), we calculated the energy surface around the BO configuration. The SIA atom was displaced in the plane defined by two lines: one connecting $\mathrm{BO}$ and the centre of basic hexagon in Fig. 1, another passing BO along the $c$ axis. This plane contains both the BO$\mathrm{M}-\mathrm{BS}$ and BO-O hops and shown by the red line rectangle in the Fig. 2. It appeared that the energy surface (see Fig. 4) has a valley along $c$ direction with the lowest point in the BO configuration. The $\mathrm{M}$ configuration is positioned on one side of this valley and the energy around it is flat within the energy grid. The shape of energy surface implies that in-plane defect oscillations will be more frequent than the out of plane. Since the oscillation frequency correlates with the jump attempt frequency, the out of plane diffusion coefficient should be even smaller than our results presented in Section VI, where an isotropic attempt frequency is assumed.

Our results show that the energy of $\mathrm{BS}$ configuration is right above those of $\mathrm{BC}^{\prime}$ and $\mathrm{M}$ and below that of $\mathrm{O}$ configuration. This agrees with the results obtained for large modelling cells using the VASP package $[19,22]$. The energy of O configuration is right above the BS. For the VASP calculations, at least a 289 atom modelling cell is needed to reproduce the right hierarchy of SIA configurations (see Table 2 and results in [19,22,23]). This difference should be attributed to the difference in pseudo-potentials used in the QE and VASP codes. The QE pseudo-potential has more compact core and gives cla ratio smaller than that from the VASP calculations, and close to the experimental value. We believe that the use of an overestimated $c / a$ ratio results in artificial preference for 'volumetric' $\mathrm{O}$ and $\mathrm{S}$ configurations, as opposed to the planar BO and BS configurations (see discussion in [20]). This difference is even larger for the 
SIESTA calculations (see Table 2), for which the c/a ratio is equal to 1.613 [17] (cf. with experimental 1.59 or $\mathrm{QE} 1.6$ ) and $\mathrm{O}$ and even the $\mathrm{S}$ configuration has a lower energy than $\mathrm{BS}$.

The PS and P2S configurations are local minima on the $\mathrm{O}$ to $\mathrm{S}$ migration path (see Fig. 5) and their formation energies obtained here are close to those in Ref. [22] (see Table 2). The stability of these configurations was verified by direct optimization of the configurations of NEB images. The absence of local minima on the migration path is due to the small number of NEB images. The PS formation energy is very close to that for $\mathrm{S}$ and is higher than for $\mathrm{O}$ configuration by $0.14 \mathrm{eV}$. The $\mathrm{P} 2 \mathrm{~S}$ configuration is $0.33 \mathrm{eV}$ higher than $\mathrm{O}$, and is the highest in energy. These three configurations correspond to "split-atoms" configurations of different orientation [22].

\section{SIA MIGRATION BARRIERS}

We start calculations of the SIA migration with the jumps from the ground BO state [1823 ] to configurations $\mathrm{O}, \mathrm{BS}$ and $\mathrm{S}$, which are closest in energy. The $\mathrm{BO}$ to $\mathrm{BS}$ jump determines in-basal-plane diffusion and the BO-O jump the out-of-basal plane migration, see Fig. 1. As shown in Fig. 3, the energy barriers of both BO-BS (shown by circles) and BO-O (rectangles) jumps are very similar, with the activation energies being equal to 0.25 and $0.27 \mathrm{eV}$, respectively. The BO-BS barrier is lower than BO-O by $0.015 \mathrm{eV}$ (see Table 3) and is close to the difference in formation energy of these two configurations, which is $-0.02 \mathrm{eV}$ (see Table 2). A recent study by Varvenne et al. [23] which included elastic corrections obtained a somewhat larger difference $(\sim 0.25$ and $\sim 0.29 \mathrm{eV}$ for BO-BS and BO-O jumps, respectively). The BO-S barrier of $0.526 \mathrm{eV}$ is two times larger than those for BO-O and BO-BS jumps. Therefore, the corresponding jumps should be suppressed at low enough temperature. Hence, the results obtained below in section VI must be a lower estimate of the diffusion anisotropy.

The BS-BS migration path represents a shift of the BS to the nearest equivalent position (see Fig. 1) with the same orientation. In the BS-BS NEB calculations, we obtained that the SIA in the BS configuration does not jump to another BS state directly but through intermediate BO configuration. Naturally, the BO jumps to another BO through BS. Below we use this combined barrier to describe BO-BS-BO and BS-BO-BS transitions. 
The next group of barriers corresponds to transitions from $\mathrm{O}$ to either $\mathrm{S}$ or BS (see Fig. 5, 6). Both energy profiles contain local energy minima. The O-BS jump passes through M configuration, which is close to BO (see Fig. 2). The SIA jumps from $\mathrm{O}$ to $\mathrm{M}$ configuration with an activation energy of $0.115 \mathrm{eV}$ and then to $\mathrm{BO}$ configuration, since the M-BO barrier of 0.008 $\mathrm{eV}$ is negligibly small. For the same reason the direct BS-O jump is also suppressed, since while passing from BS through the $\mathrm{M}$ configuration the system goes directly to the BO configuration. The O-S transition is even more complicated and contains four intermediate states (see Fig. 5). The SIA first jumps from $\mathrm{O}$ to $\mathrm{BC}^{\prime}$ configuration, the latter being close to $\mathrm{M}$ configuration (see section III); and, then reaches S configuration via a series of "split atoms" configurations (PSP2S-S, connected by rotations about common center of gravity). Thus, the O-BC' jump barrier was assumed to be equal to that of the O-M, which was calculated with a larger number of intermediate images. As can be seen from the energy profile presented in Fig. 4, the BO, M and $\mathrm{BC}^{\prime}$ configurations are in a flat valley with the $\mathrm{BO}$ configuration in the minimum. From the $\mathrm{BC}^{\prime}$

(or $\mathrm{M}$ ) configuration the system passes athermally to the BO configuration. Thus, even though the NEB technique does not directly show it, the migration path from $\mathrm{O}$ to $\mathrm{S}$ includes the intermediate $\mathrm{BO}$ configuration. A more detailed description of the $\mathrm{BC}^{\prime}$ to $\mathrm{S}$ transition was not attempted here. As we mentioned in Section III, the metastability of the PS, PS' and P2S configurations was verified by direct relaxation of corresponding NEB images.

Finally the BS to S rotation was studied. As can be seen from Fig. 7, the system passes through some intermediate states, and the migration barrier was found to be $0.375 \mathrm{eV}$. We note that the out of plane movement from BS to $\mathrm{O}$ configuration requires another $0.275 \mathrm{eV}$, which makes the relative probability of such a transition negligible at temperatures of practical importance. For this reason, details of this transition were not studied. .

\section{VACANCY MIGRATION BARRIERS}

First principle results on the vacancy migration barriers were obtained by Verite et al. [17] using a simulation box of $3 \times 3 \times 2$ unit cells. The barriers were found to be equal to $0.51 \mathrm{eV}$ for basal plane jumps and $0.67 \mathrm{eV}$ for non-basal jumps. Both barriers were "single humped", while the shape of the basal energy barrier around its maximum was found to be very flat. We used 
$4 \times 4 \times 3$ unit cells in our calculations. An increase of the cell size leads to only slight modification of the barrier for a basal plane jump (see Fig. 8). The barrier was found to have a "double humped" shape with a shallow minimum at its central point. We believe that this minimum is caused by larger "space" for atomic relaxation in bigger simulation cell. The energies were found to be $0.538 \mathrm{eV}$ for the basal and $0.629 \mathrm{eV}$ for non-basal jumps.

\section{MONTE CARLO SIMULATIONS OF DEFECT DIFFUSION}

Point defect diffusion was simulated as a Markovian process of defect jumps on the lattice, using the 'residence time algorithm' [36]. For a defect at a site $i$, the probability of jump to site $j$ is

$$
p_{i j}=v_{i j} \tau_{i}
$$

where $\tau_{i}=\left(\sum_{k} v_{i k}\right)^{-1}$ is the mean time delay before jumps, and

$$
v_{i j}=v_{0} \exp \left(-E_{i j} / k T\right)
$$

is the defect jump frequency from site $i$ to site $j, E_{i j}$ is the activation energy associated with this jump, $v_{0}$ is the attempt frequency, which is assumed to be independent of the jump type, and $k T$ has the usual meaning of the thermal energy. The jump direction was chosen according to the probability distribution given by Eq. (1) and, for any jump, the mean time delay before the jump, $\boldsymbol{\tau}_{i}$, was added to the total time, $t$, of the trajectory. The defect displacement vectors, $\mathbf{R}$, were recorded during simulations and used to estimate the diffusion coefficients in the $c$-axis direction and for basal plane migration ( $D_{\mathrm{c}}$ and $D_{\mathrm{a}}$, respectively) using the Einstein equation:

$$
D_{\alpha}=\frac{\left\langle\mathbf{R}_{\alpha}^{2}\right\rangle}{2 n t},
$$

where $n=1$ for $\alpha=c$ (1-D diffusion) and $n=2$ for $\alpha=a$ (2-D diffusion). In this equation, the triangle brackets denote averaging over different trajectories, and $\mathbf{R}_{\mathrm{c}}$ and $\mathbf{R}_{\mathrm{a}}$ are the projections of the displacement vector on the $c$ axis and basal plane, respectively. Approximately 300,000 trajectories of $\sim 1000$ jumps each were generated for each particular temperature, so that the statistical errors were negligible. 
The diffusion scenario for vacancies consisted of two jumps, one basal and one non-basal, described in Section V above. For the SIAs, three configurations were considered, the BS, BO and $\mathrm{O}$, while the $\mathrm{M}$ configuration was omitted, since both energetically and spatially it is very similar to the BO configuration. The ab initio data on the energy barriers for the SIA jumps in Table 3 and $\mathrm{c} / \mathrm{a}=1.60$ were used. BS configurations with three different axis orientations were distinguished, and two jumps from each such BS configuration to the $\mathrm{BO}$ lying in the same atomic triangle were permitted. Thus, six BO-BS, four BS-BO, two BO-O and two O-BO jumps were considered.

The calculated temperature dependence of the diffusion anisotropy, i.e. the ratio $D_{\mathrm{a}} / D_{\mathrm{c}}$, for SIAs and vacancies were calculated and are presented in Fig. 9 for the temperature range from 200 to $1100 \mathrm{~K}$ and compared with available experimental data for vacancy diffusion [10,11]. Both vacancies and interstitials are observed to exhibit anisotropic diffusion, with the diffusion anisotropy higher for vacancies than SIAs for temperatures up to $\sim 900 \mathrm{~K}$. At higher temperatures, the SIA diffusion becomes more anisotropic but this temperature range is not as important for practical applications. Note that the experimental value at $1000 \mathrm{~K}$ occasionally coincides with the calculations for vacancies; however, the experimental errors were rather large, which is indicated by significantly lower value for $1100 \mathrm{~K}$.

The result obtained is somewhat unexpected. In particular, the DAD model [8] proposed for the description of radiation growth in $\mathrm{Zr}$ is based on the assumption that the SIA diffusion is anisotropic whereas vacancy migration is isotropic. Radiation growth is described in terms of the bias factors, $B_{\mathrm{d}}$, which characterize the preference of mobile defects for absorption by various dislocation-type defects. The bias factors derived in [8] are given by

$$
B_{\mathrm{d}}= \begin{cases}1-\frac{p^{2}}{1+B_{\mathrm{e}}}, & a \text {-type dislocation, } \\ 1-\frac{p^{-1}}{1+B_{\mathrm{e}}}, & c \text { - type dislocation, }\end{cases}
$$

where $p=\left(D_{\mathrm{a}} / D_{\mathrm{c}}\right)^{1 / 6}$ and $B_{\mathrm{e}}$ is the elastic bias factor, which is due to the so-called size effect, i.e. the difference in interaction of vacancy and SIA with the dislocation stress field. It was shown in [8] that the diffusion anisotropy may explain radiation growth in Zr. As shown above, the 
situation is more complicated, since vacancy diffusion is also anisotropic, and the total bias should be a difference of interstitial and vacancy contributions

$$
B \text { deff }=B d-B d v
$$

where $B_{\mathrm{d}}$ is given by Eq. (4) with the diffusion anisotropy ratio $p$ for SIAs and with $B_{\mathrm{e}}=0.10$ (the value used in [8]), whereas $B_{\mathrm{d}}^{\mathrm{v}}$ is given by Eq. (4) with the $p$ for vacancies and $B_{\mathrm{e}}=0$ to account for the effect of anisotropy only.

The temperature dependence of the effective bias factor, $B_{\mathrm{d}}^{\text {eff }}$, for different dislocation sinks calculated using Eq. (5) and data from Fig. 9 is presented in Fig. 10. There is a crossover temperature of $\sim 750 \mathrm{~K}$; below this temperature the bias factors are negative for dislocations with $a$-type Burgers vectors. With these data, the DAD theory fails to describe radiation growth in $\mathrm{Zr}$ below $\sim 750 \mathrm{~K}$, where positive strains are observed in $a$ directions and negative strains in $c$ directions. Indeed, the positive $a$ strain (as well as the growth of the $a$-type interstitial loops and $c$-type vacancy loops) is provided by an excess of SIAs arriving at prismatic $a$ dislocations due to preferential diffusion of SIAs along basal planes. Our results show more significant anisotropy of vacancy than SIA diffusion along the basal planes, which produces negative $a$ strain. This result can be understood based on the following experimental observation. It is well known that the density of $c$ dislocations is significantly smaller than $a$ dislocations in Zr-based alloys. In this case, the combined effect of $a$ and $c$ dislocations in accumulation of strain should be determined by the bias factor for $a$-type dislocation defects, and $c$ dislocations would not contribute significantly.

In addition, this result contradicts the observed aligning of vacancy loops and voids $[12,38]$ along $c$ planes. As mentioned in the Introduction section, according to Foreman [13], the void alignment is associated with the increasing the mutual screening of defects from mobile interstitial defects, whereas our results predict net SIA flux along $c$ direction.

The implied contradiction between theory and experiment can be resolved by postulating the formation of small SIA clusters during $1 \mathrm{MeV}$ electron irradiation, which would migrate preferentially within the basal planes. This would significantly increase the effective diffusion 
anisotropy of SIAs and make the reaction-diffusion scenario much closer to that under neutron irradiation conditions, where small SIA clusters are formed in cascades of atomic displacements.

\section{CONCLUSIONS}

A combination of DFT methods, kinetic Monte Carlo and rate theory was applied to analyze point defect migration in hcp $\mathrm{Zr}$ and their effect on the observed radiation growth of $\mathrm{Zr}$ under electron irradiation. The following conclusions were drawn.

1. The size of the DFT simulation cell in the $a$ direction must be at least $6 a$ to correctly describe hierarchy of different SIA configurations.

2. SIA migration may be considered jumps between $\mathrm{BO}, \mathrm{BS}$ and $\mathrm{O}$ configurations. Other configurations, $\mathrm{M}$ and $\mathrm{BC}$, are energetically and spatially close to $\mathrm{BO}$, and may be considered to be an extension of the BO configuration.

3. In-basal-plane jumps are the most favorable for both vacancies and SIAs, so that both defects exhibit anisotropic diffusion, predominantly parallel to the basal planes.

4. The difference in the in-basal and out-of-basal energy barriers for SIAs for is $\sim 0.015 \mathrm{eV}$, so that the temperature dependence of the SIA diffusion anisotropy is weak.

5. The same difference for vacancies is $\sim 0.09 \mathrm{eV}$, which provides stronger temperature dependence for the vacancy diffusion anisotropy.

6. The diffusion anisotropy is higher for vacancies than SIAs for temperatures up to $\sim 900 \mathrm{~K}$ (Fig. 9). At higher temperatures, the SIA diffusion becomes more anisotropic.

7. The diffusion anisotropy of point defects obtained cannot explain the observed positive strains in prismatic and negative strains in basal directions in $\mathrm{Zr}$. It is speculated that this contradiction may be resolved by the formation of small SIA clusters with more significant anisotropy of diffusion.

\section{ACKNOWLEDGEMENTS}

This research was supported by the Consortium for Advanced Simulation of Light Water Reactors, an Energy Innovation Hub (http://www.energy.gov/hub) for Modelling and Simulation 
of Nuclear Reactors under U.S. Department of Energy Contract No. DE-AC05-00OR22725, and used resources of the National Energy Research Scientific Computing Center, which is supported by the Office of Science of the U.S. Department of Energy. 


\section{REFERENCES}

[1] S.I. Golubov, A.V. Barashev, R.E. Stoller, ORNL Report: ORNL/TM-2011/473, Oak Ridge National Laboratory, 2011.

[2] S.I. Golubov, A.V. Barashev, R.E. Stoller, B.N. Singh, "Breakthrough in Understanding Radiation Growth of Zirconium," Zirconium in the Nuclear Industry, 17th International Symposium, ASTM STP 1543, R.J. Comstock, Ed., ASTM International, West Conshohocken, PA, (in press).

[3] S.J. Wooding, L.M. Howe, F. Gao, A.F. Calder, D.J. Bacon, J. Nucl. Mater., 1998; 254;191.

[4] R.E. Voskoboinikov, Yu.N. Osetsky and D.J. Bacon, Journal of ASTM International, 2005; 2; JAI12410.

[5] R.E. Voskoboinikov, Yu.N. Osetsky and D.J. Bacon, Nucl. Instr. And Meth. Phys. Res. B, $2006 ; 242 ; 68$.

[6] N. de Diego, Yu. N. Osetsky and D.J. Bacon, Metall. Transactions, Physical Metallurgy and Materials Sci., 2002; 33A; 783.

[7] C.H. Woo, U. Gösele, J. Nucl. Mater., 1983; 119; 219.

[8] C.H. Woo, J. Nucl. Mater., 1988; 159; 237.

[9] Yu. N. Osetsky, D. J. Bacon, N. De Diego, Metallurgical and Materials Transactions, 2002; 33A; 777.

[10] G.M. Hood, Defects and Diffusion Data, 1993; 95-98; 755.

[11] G.M. Hood, H. Zou, R.J. Schultz, N. Matsuura, J.A. Roy, and J.A. Jackman, Def. Diff. Forum, 1997; 143-147; 49.

[12] Y. de Carlan, C. Regnard, M. Griffiths, D. Gilbon, C. Lemaignan, ASTM STP, 1996; 1295; 638.

[13] A.J.E. Foreman, Report No. AERE-R-7135, UKAE Authority, Harwell, 1972.

[14] A.V. Barashev, S.I. Golubov, Phil. Mag., 2010; 90; 1787.

[15] F. Willaime, J. Nucl. Mater., 2003; 323; 205.

[16] C. Domain, A. Legris, Phil. Mag., 2005; 85; 569.

[17] G. Verite, F. Willaime, C.C. Fu, Solid State Phenomena, 2007; 129; 75. 
[18] G.D Samolyuk, S.I. Golubov, Y.N. Osetsky, R. E. Stoller, ORNL/TM-2011/516, Oak Ridge National Laboratory, 2012.

[19] Q. Peng, W. Ji, H. Huang, S. De, J. Nucl. Mater., 2012; 429; 233.

[20] G.D Samolyuk, S.I. Golubov, Y.N. Osetsky, R.E. Stoller, Phil. Mag. Lett., 2013; 93; 93.

[21] Q. Peng, W. Ji, H. Huang, S. De, J. Nucl. Mater., 2013; 437; 293.

[22] G. Vérité, C. Domain, Chu-Chun Fu, P. Gasca, A. Legris, F. Willaime, Phys. Rev. B, 2013; $87 ; 134108$.

[23] C. Varvenne, F. Bruneval, M.-C. Marinica, E. Clouet, Phys. Rev. B, 2013; 88; 134102.

[24] P. Giannozzi, S. Baroni, et al., J. Phys. Condens. Matter., 2009; 21; 395502.

[25] J.P. Perdew, K. Burke, M. Ernzerhof, Phys. Rev. Lett., 1996; 77; 3865.

[26] D. Vanderbilt, Phys. Rev. B, 1990; 41; 7892.

[27] We used the pseudopotential Zr.pbe-nsp-van.UPF from: http://www.quantum-espresso.org.

[28] G. Kresse, J. Hafner, Phys. Rev. B, 1993; 47; 558, ibid. 1994; 49; 14251.

[29] J.P. Perdew, Y. Wang, Phys. Rev. B, 1992; 45; 13244.

[30] H. Jonsson, G. Mills, K. W. Jacobsen, Classical and Quantum Dynamics in Condensed Phase Simulations, B. J. Berne, G. Ciccotti, D. F. Coker, eds. (World Scientific, Singapore, 1998) pp. 385-404.

[31] G. Henkelman, B. P. Uberuaga, H. Jonsson, J. Chem. Phys., 2000; 113; 9901.

[32] G. Henkelman, H. Jonsson, J. Chem. Phys., 2000; 113; 9978.

[33] C. Domain, R. Besson, A. Legris, Acta Mater., 2002; 50; 3513.

[34] L. Fast, J.M. Wills, B. Johansson, O. Erikson, Phys. Rev. B, 1995; 51; 17431.

[35] J.P. Perdew and A. Zunger (1981). Phys. Rev. B, 1981; 23; 5048.

[36] W.M. Young, E.W. Elcock, Proc. Phys. Soc., 1966; 89; 735.

[37] E. Fisher, C. Renken, Phys. Rev. A, 1964; 135; 482.

[38] M.G. Griffiths, R.A. Holt, A. Rogerson, J. Nucl. Mater., 1995; 225; 245. 


\section{FIGURE CAPTIONS}

Fig. 1. Typical configurations of SIA in the hep lattice and calculated diffusion paths shown by arrows connecting two corresponding configurations.

Fig. 2. M configuration of SIA. The perfect crystal prism is shown by solid lines where atoms are placed in the corners. The positions of the atoms in the basal plane after introduction of SIA in $\mathrm{M}$ configuration are shown by grey circles. The region around $\mathrm{BO}$ containing both $\mathrm{BO}$ and $\mathrm{M}$ configurations is shown by the red rectangle.

Fig. 3. The energy profiles for SIA migration starting from the BO state as a function of the reaction coordinate, the results for the BO-S jump are shown by blue up-triangles, BO-O by green squares, $\mathrm{BO}-\mathrm{BS}$ by red circles and $\mathrm{BO}-\mathrm{M}$ by grey down-triangles.

Fig. 4. The energy surface calculated for SIA configuration close to BO. The SIA atom was displaced in the rectangle region shown by red lines in Fig. 2, which contains the BO, M and BC' configurations. The surface was produced using spline interpolation for the energy points calculated on a 20 point grid.

Fig. 5. The O-S migration energy barrier, calculated by NEB method, corresponds to jumps through a few intermediate metastable configurations. According to the result presented in Fig. 4, the $\mathrm{M}-\mathrm{BC}^{\prime}$ jump does not have a barrier. .

Fig. 6. The O-BS energy barriers as a function of reaction coordinate is shown by purple points connected by lines. The intermediate configuration $(\mathrm{M})$ is shown by diamond.

Fig. 7. The BS-S migration path energy barrier is shown by red points connected by line.

Fig. 8. The vacancy migration energy barriers: basal and non-basal shown by blue and red color respectively.

Fig. 9. Temperature dependence of the diffusion anisotropy factors for SIAs and vacancies together with two experimental points from $[10,11]$.

Fig. 10. Temperature dependence of the bias factors of dislocation-type defects calculated for $B_{\mathrm{e}}=0.1$. 


\section{LIST OF TABLES}

1. Bulk properties of hep Zr: lattice parameter $a(\AA), c / a$ and elastic constants $\left(C_{i j}\right)(\mathrm{GPa})$. The calculated results are for different exchange-correlation functional (XC). The $C_{i j}$ values in parenthesis denote the results without internal relaxation.

2. The calculated defect formation energy $\Delta E_{\mathrm{j}}=E_{\mathrm{j}}-E_{\mathrm{O}}(\mathrm{eV})$ for super-cells containing $\mathrm{N}$ atoms and different exchange-correlation functional (XC).

3. Migration energy barriers (eV) and corresponding jump length $(\AA)$. 
Table 1. Bulk properties of hep Zr: lattice parameter $a(\AA)$, cla ratio and elastic constants $\left(C_{i j}\right)$ (GPa). The results are for different exchange-correlation functional (XC). The $C_{i j}$ values in parenthesis calculated without internal relaxation.

\begin{tabular}{|c|c|c|c|c|c|c|c|c|c|c|}
\hline Ref. & $\mathrm{XC}$ & $a$ & $c / a$ & $B$ & $C_{11}$ & $C_{12}$ & $C_{13}$ & $C_{33}$ & $C_{44}\left(C_{55}\right)$ & $\begin{array}{c}C_{66}=\left(C_{11}\right. \\
\left.C_{12}\right) / 2\end{array}$ \\
\hline $\mid$ Exp. [37] & & 3.23 & 1.593 & 97 & 155 & 67 & 65 & 173 & 36 & 44 \\
\hline [15] & $\begin{array}{c}\text { LDA PZ } \\
{[35]}\end{array}$ & 3.15 & 1.615 & 105 & 135 & & & 181 & 21 & 24 \\
\hline [33] & $\begin{array}{c}\text { LDA PZ } \\
{[35]}\end{array}$ & 3.16 & 1.614 & 105 & 145 & & & 177 & 22 & 36 \\
\hline [34] & LDA & 3.19 & 1.585 & 103 & (156) & (65) & (76) & 182 & 25 & (46) \\
\hline [15] & $\begin{array}{c}\text { GGA PBE } \\
{[25]}\end{array}$ & 3.23 & 1.600 & 94 & $\begin{array}{c}146 \\
(153)\end{array}$ & & & 156 & 28 & $42(49)$ \\
\hline [33] & $\begin{array}{c}\text { GGA PW } \\
{[29]}\end{array}$ & 3.23 & 1.604 & 92 & 142 & & & 164 & 29 & 39 \\
\hline $\begin{array}{c}\text { Present } \\
\mathrm{QE}\end{array}$ & $\begin{array}{c}\text { GGA PBE } \\
{[25]}\end{array}$ & 3.23 & 1.600 & 95 & $\begin{array}{c}140 \\
(151)\end{array}$ & $\begin{array}{c}69 \\
(58)\end{array}$ & 68 & 166 & 26 & $36(47)$ \\
\hline
\end{tabular}


Table 2. The calculated defect formation energy $\Delta E_{\mathrm{j}}=E_{\mathrm{j}}-E_{\mathrm{O}}(\mathrm{eV})$ for super-cells containing $\mathrm{N}$ atoms and different exchange-correlation functional (XC).

\begin{tabular}{|l|c|c|c|c|c|c|c|c|c|c|c|c|}
\hline \multirow{2}{*}{$\mathrm{XC}$} & & $\mathrm{N}$ & $E_{\mathrm{O}}$ & $\Delta E_{\mathrm{S}}$ & $\Delta E_{\mathrm{BO}}$ & $\Delta E_{\mathrm{BS}}$ & $\Delta E_{\mathrm{M}}$ & $\Delta E_{\mathrm{BC}}$ & $\Delta E_{\mathrm{PS}}$ & $\Delta E_{\mathrm{PS}}$ & $\Delta E_{\mathrm{P} 2 \mathrm{~S}}$ & $\Delta E_{\mathrm{C}^{\prime}}$ \\
\hline GGA & QE & 145 & 2.92 & 0.10 & -0.15 & -0.02 & -0.08 & -0.10 & 0.06 & 0.14 & 0.33 & 0.14 \\
\hline GGA & VASP & 145 & 2.80 & 0.18 & -0.07 & 0.05 & -0.06 & & & & & \\
\hline GGA & SIESTA [22] & 289 & 3.16 & 0.04 & -0.13 & 0.10 & & -0.04 & & 0.12 & 0.27 & 0.11 \\
\hline GGA & VASP [22] & 201 & 2.87 & 0.14 & -0.09 & 0.02 & & -0.06 & & 0.14 & 0.35 & 0.13 \\
\hline GGA & VASP [19] & 289 & 2.92 & 0.16 & -0.14 & -0.03 & & & & & & \\
\hline
\end{tabular}

Table 3. Migration energy barriers $(\mathrm{eV})$ and corresponding jump length $(\AA)$.

\begin{tabular}{cccc}
\hline Transition & Forward $(\mathrm{eV})$ & Backward $(\mathrm{eV})$ & Distance $(\AA)$ \\
\hline BO-BS & 0.254 & 0.124 & 1.865 \\
BO-O & 0.269 & 0.123 & 1.292 \\
BO-S & 0.526 & 0.275 & 3.187 \\
BO-M & 0.073 & 0.008 & 0.748 \\
O-M & 0.115 & 0.196 & 1.834 \\
M-BS & 0.150 & 0.086 & 2.010 \\
& & & \\
\hline
\end{tabular}




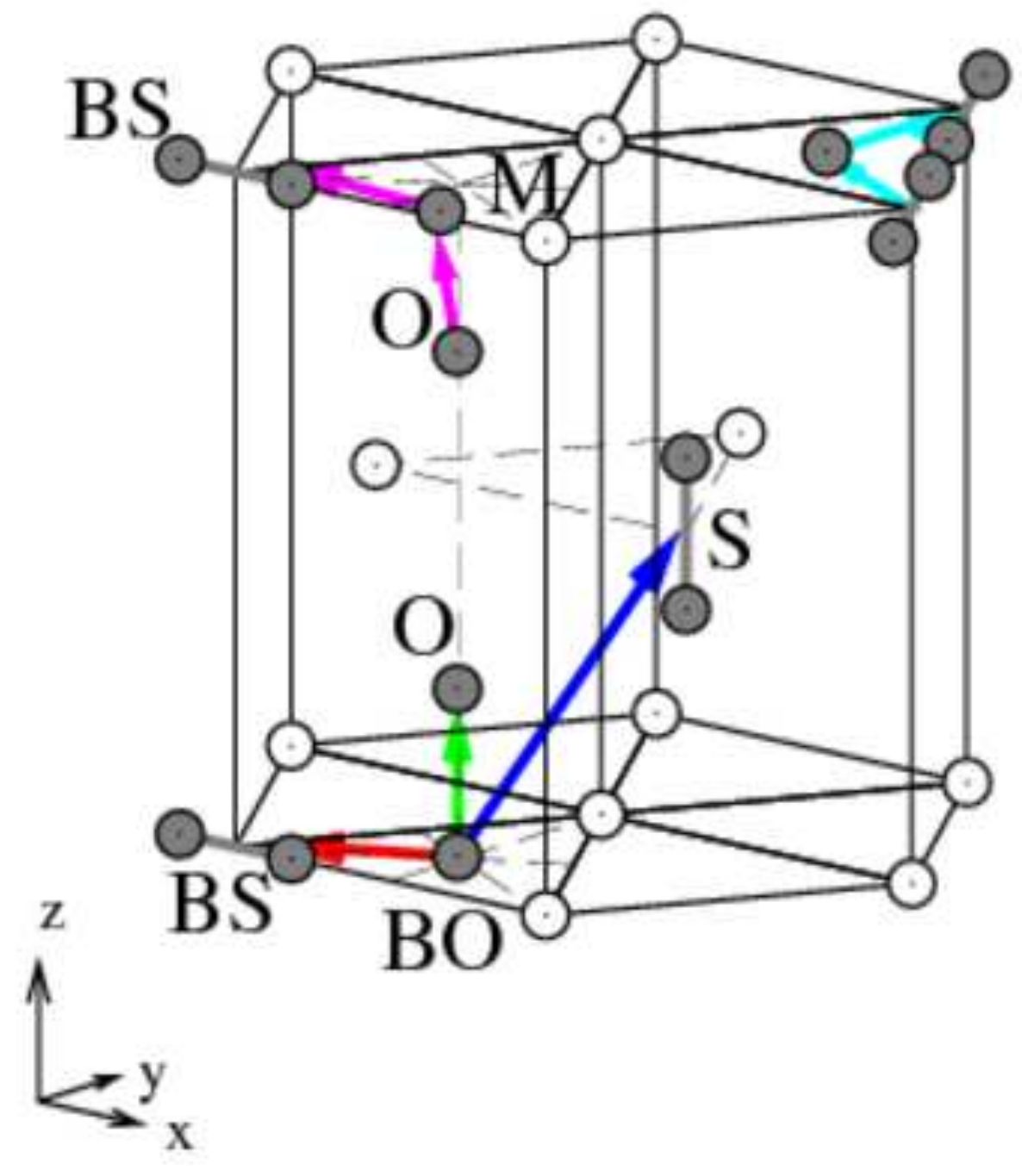


M
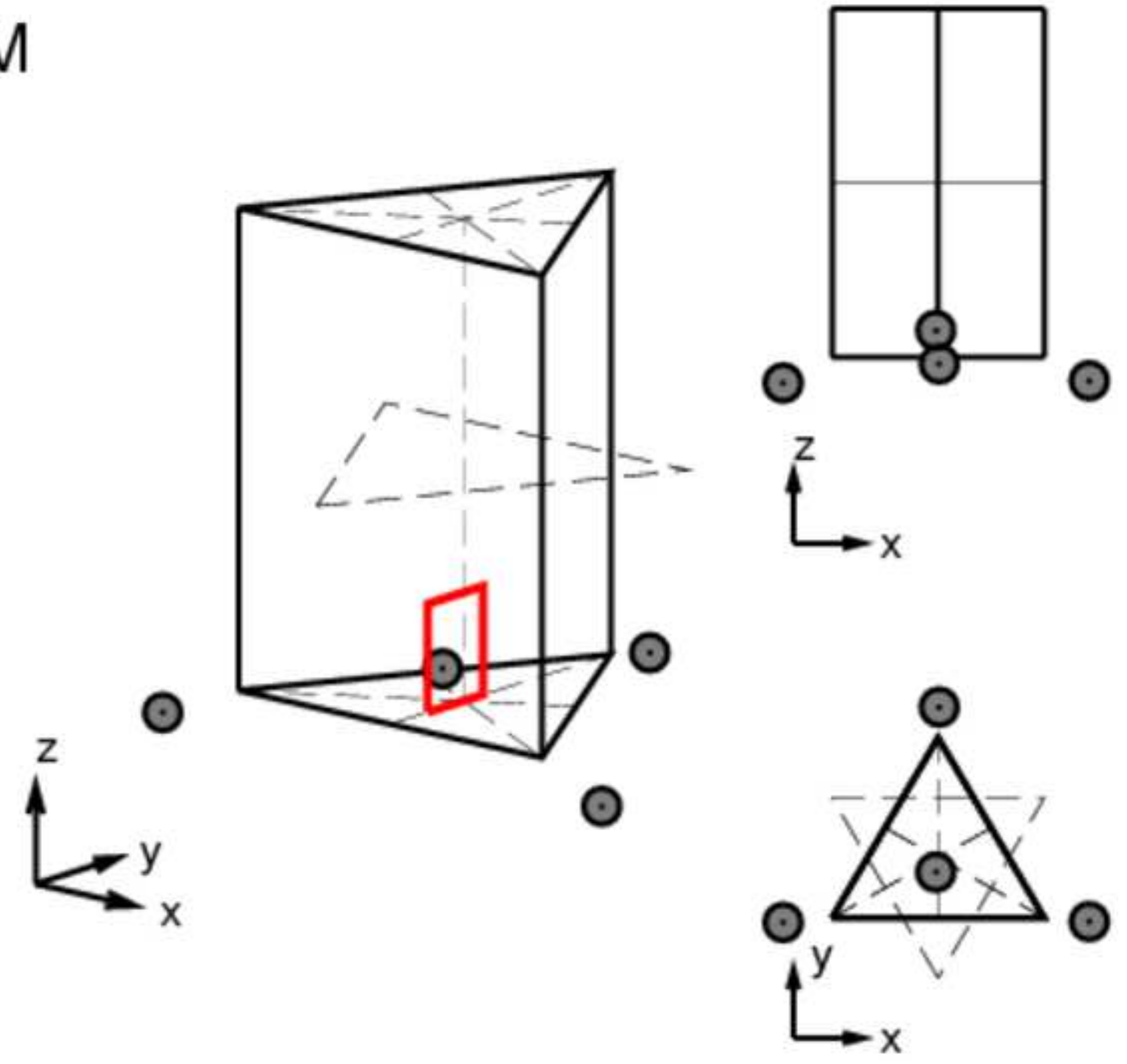


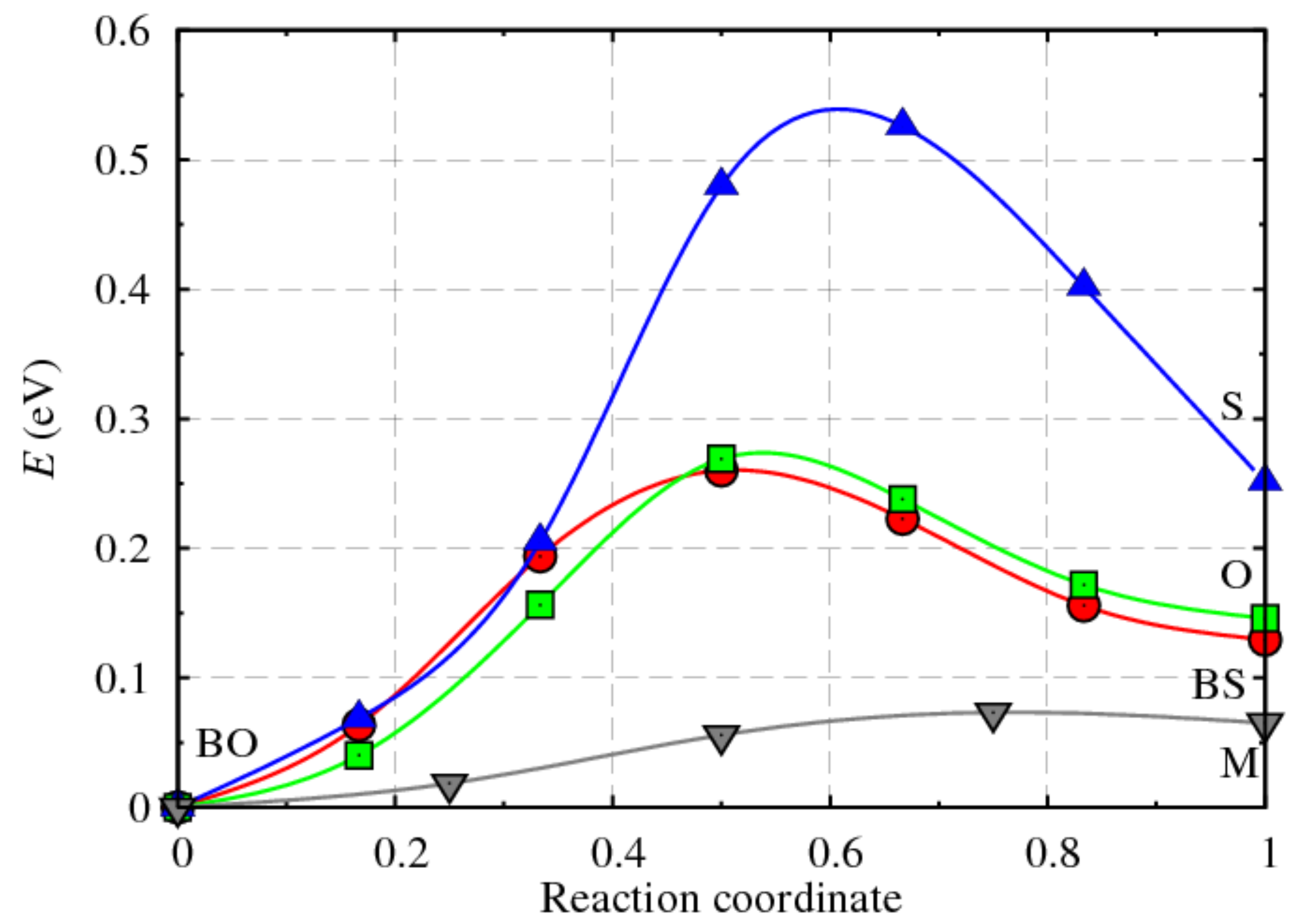




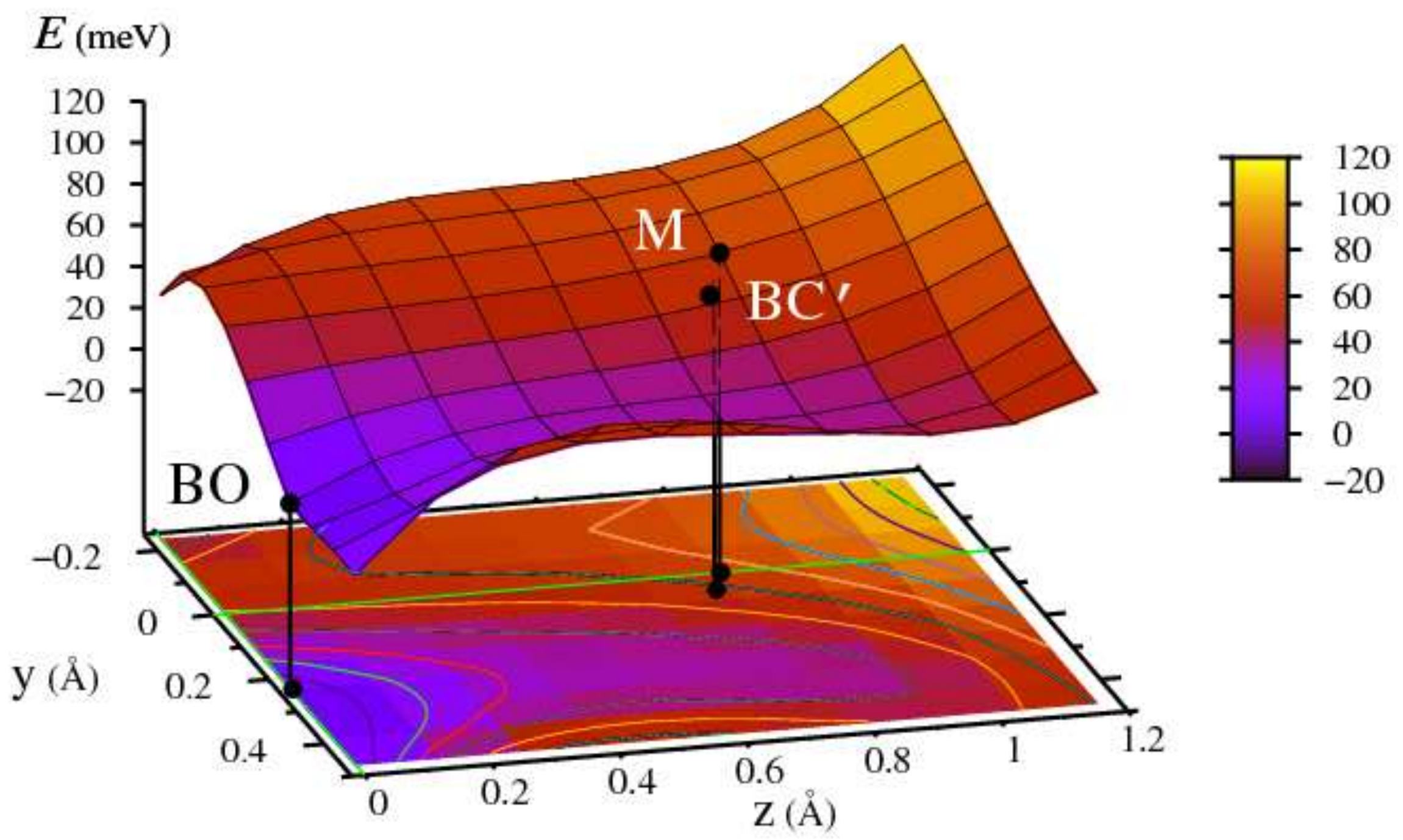




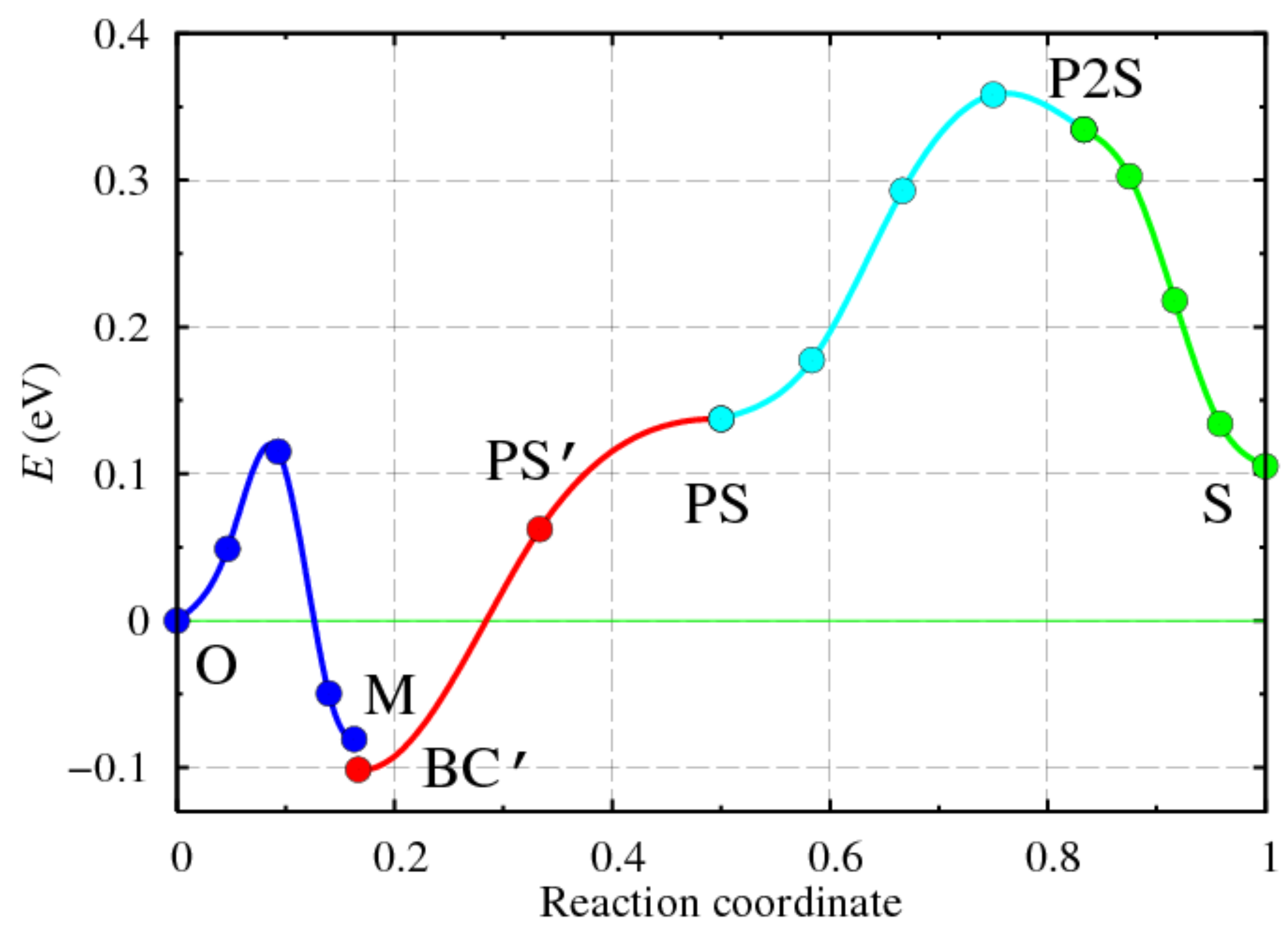




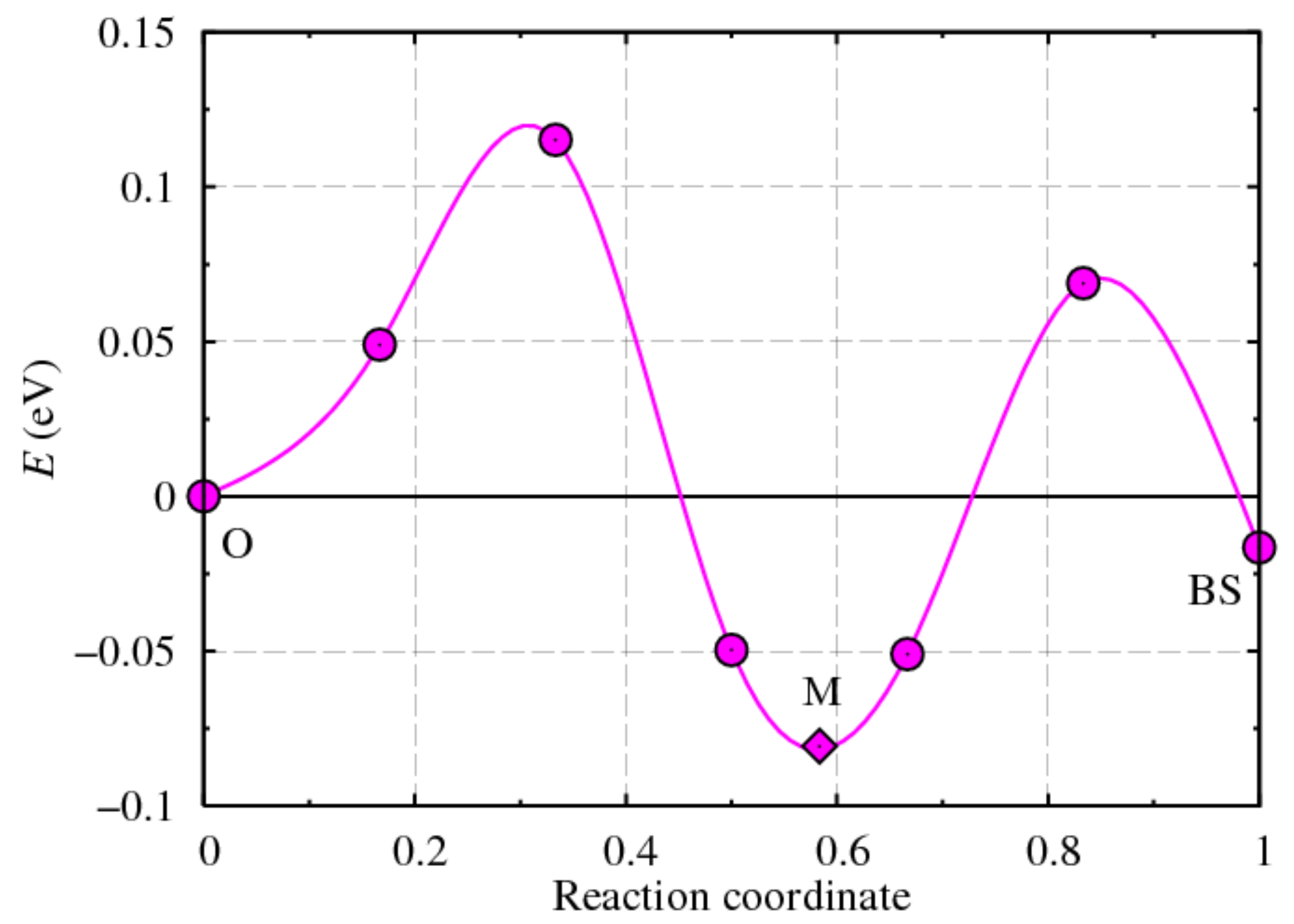




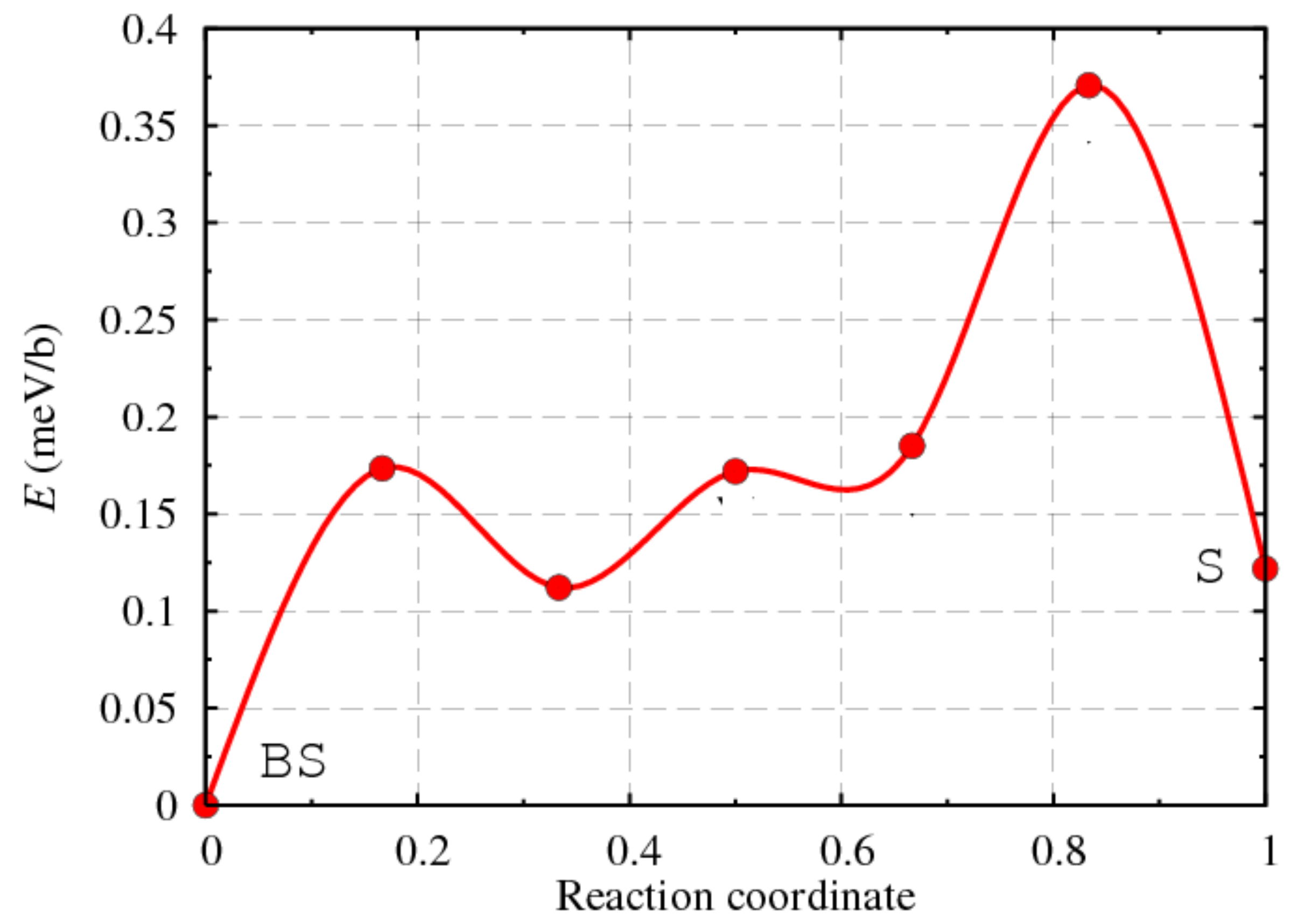




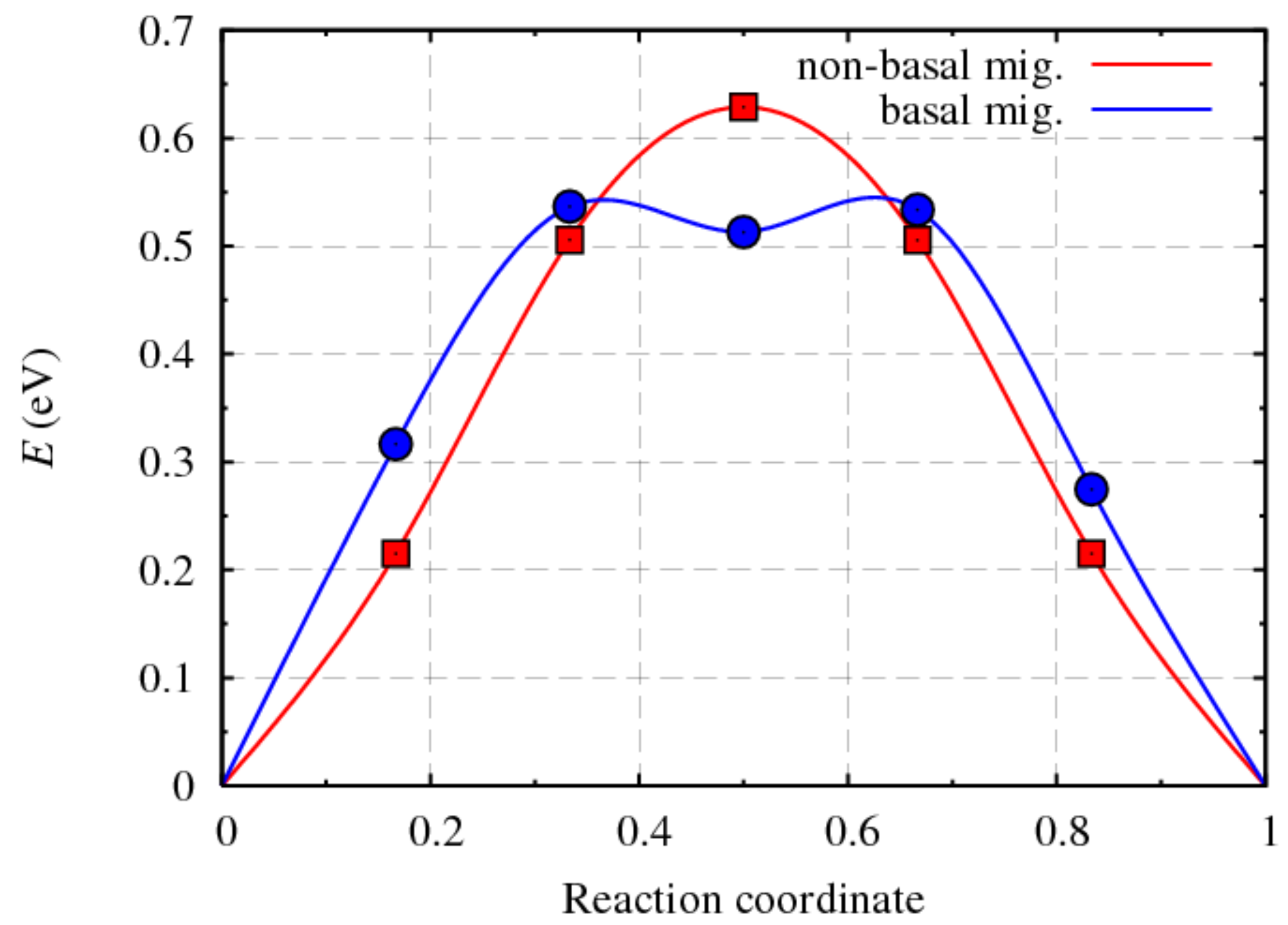




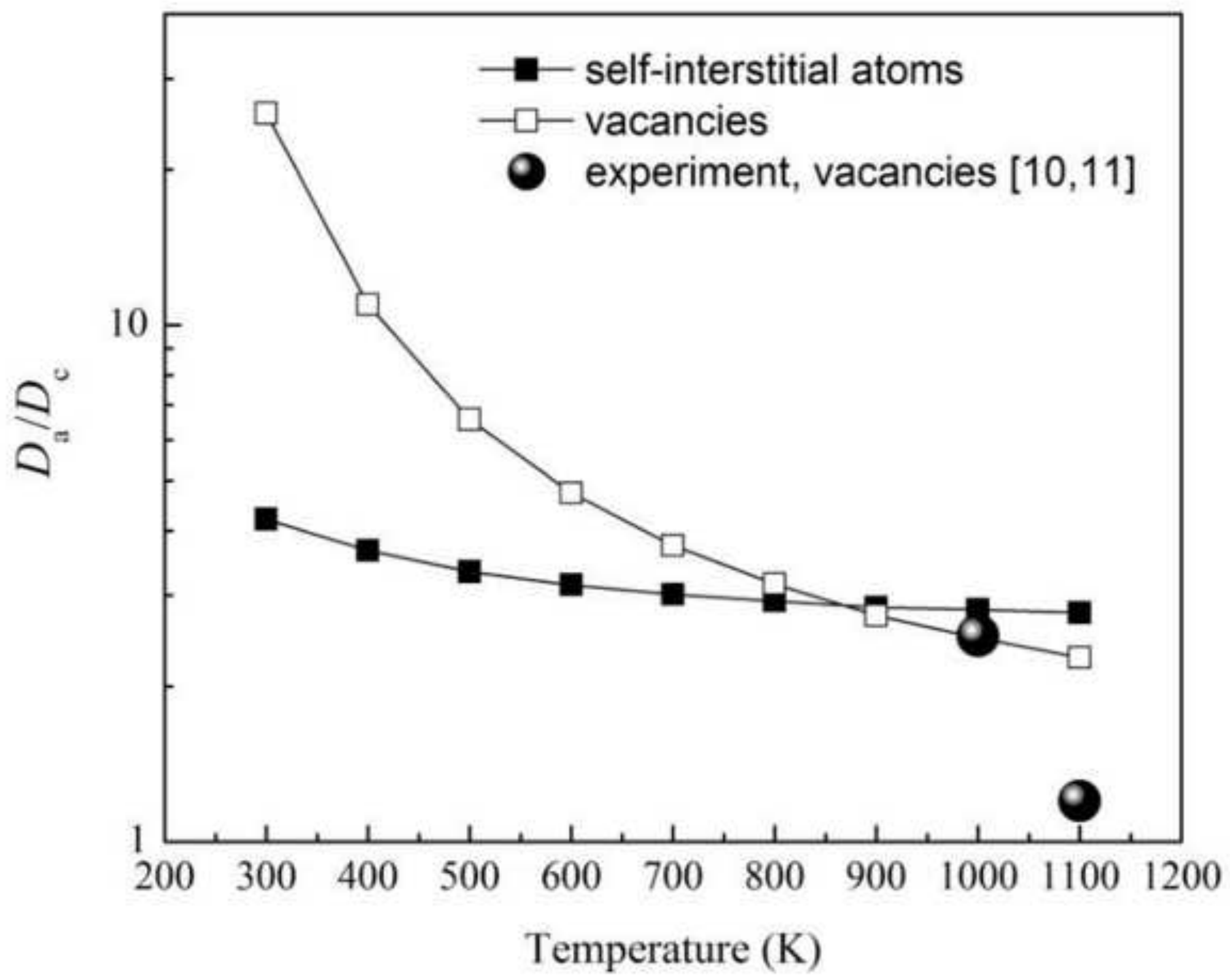




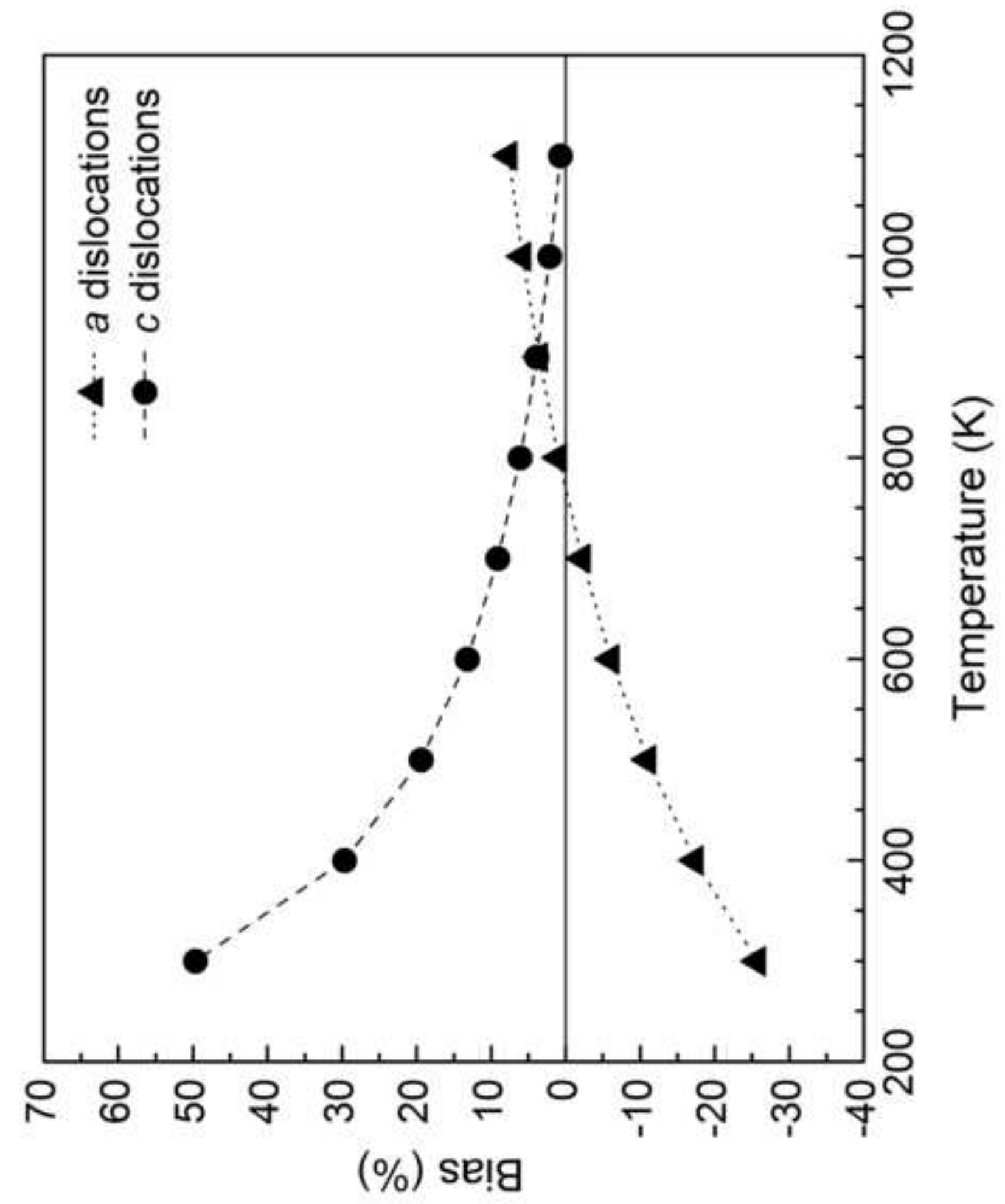

Figure(s) 